\title{
Modelos de regulación profesional de los médicos en América Latina: elementos teóricos para su análisis
}

\author{
Models for physicians' professional regulation \\ in Latin America and theoretical elements \\ for its analysis
}

Gustavo Nigenda 1

Maria Helena Machado 2

\footnotetext{
1 Centro de Investigación en Sistemas de Salud, Instituto Nacional de Salud Pública. Avenida Universidad 655, Stạ Ma Ahuacatitlan, 62508, Cuernavaca, Morelos, México.

2 Escola Nacional de Saúde Pública, Fundação Oswaldo Cruz. Rua Leopoldo Bulhões, 1480, sala 702 ,

Rio de Janeiro, RJ 21041-210, Brasil.
}

Abstract This paper reviews regulation of medical work based on concepts available in the specialized literature. It proposes the model devel oped by Moran and Wood to anal yze the regulatory process in the Latin American context. However, any conceptual model used for the analysis of regulation should consider the changes generated by the reform in the interaction between financial and regulatory agents and health care providers. The regulatory function is involving an increasing number of groups interested in the appropriate performance of health services. Regulation of medical work is a dynamic issue requiring close analysis.

Key words Health Occupations; Physicians; Health Care Reform; Health Manpower

Resumen El presentetrabajo discute el fenómeno de la regulación del trabajo médico utilizando conceptos disponi bles en la literatura especial izada. Se propone el model o desarrollado por Moran y Wood como un buen punto de partida para esta discusión en el ámbito de América Latina. Sin embargo, se consi dera que cualquier model o conceptual utilizado para la comprensión del fenómeno debe consi derar los cambios generados por la reforma en la interacción entre los agentes financieros y reguladores con los prestadores de servicios. La función regulatoria es cada dia un fenómeno que involucra más grupos interesados en el funcionamiento adecuado de los servicios de salud. La regulación del trabajo profesional es un fenómeno dinámico que requiere ser analizado de forma cercana.

Palabras clave Profesión Médica; Médicos; Reforma Sanitária; Recursos Humanos en Salud 


\section{Introducción}

El presente trabajo revisa y discute aspectos recientes sobre los cambios en los modelos de regulación del ejercicio profesional médico en perspectiva teórica. Con ello se busca identificar la forma en la que estos modelos permiten entender el papel de los profesionales en la producción de servicios de la salud tanto en su carácter de agentes económicos como de actores con interés político.

En la producción de servicios de salud participan distintos agentes económicos. A principios del siglo, en muchos países, se identificaban básicamente dos: uno era el productor y el otro el receptor de los servicios. Los agentes terciarios eran prácticamente inexistentes. En los hechos esto se traducía en estrecha relación terapeuta-paciente tanto en términos clínicos como financieros. Este tipo de práctica caracterizó a lo que comunmente se le conoce como medicina liberal. En la ideología de los médicos liberales el pago directo al médico garantizaba una relación terapéutica apropiada en donde el paciente podía confiar plenamenteen la capacidad del primero para la solución de sus problemas de salud (Wilding, 1982).

Con el tiempo y debido a múltiples circunstancias relacionadas a la búsqueda por racionalizar la provisión de servicios de salud, los mecanismos para producir y financiar dichos servicios se volvieron más complejos y ello permitió la participación de nuevos agentes. Entre los nuevos agentes se encontraba el Estado que, además de convertirse en financiador importante, inició una participación sobresaliente tanto en aspectos administrativos como regulatorios (Narro \& Moctezuma, 1992). No obstante, a pesar de que la esfera de influencia del Estado como financiador y productor de servicios se vió enormemente ampliada a partir de los años 40, prácticamente en todos los países de la América Latina se protegió un espacio para la medicina privada. En este ámbito otros agentes distintos al Estado encontraron terreno para desarrollar su participación. El papel del Estado como regulador de los grupos profesionales sin embargo se mantuvo, tanto en el ámbito público como en el privado (Academia Nacional de Medicina, 1993).

A pesar de la magnitud de las transformaciones sucedidas en la actualidad en la producción de servicios de salud, los médicos mantienen un papel predominante como agentes productores. Uno de los cambios más importantes en ese processo, en el presente siglo, fue la aparición de unidades productoras caracterizadas por su alta complejidad organizacional. Una vez in- corporados a esas unidades, los médicos fueron capaces de jugar un papel importante en la definición de las estrategias de atención, en la distribución de los recursos disponibles y en la toma de decisiones técnicas. Todo ello en función de las características de su formación técnica y de su carácter de profesionales. El estatus profesional gestado desde el período de la medicina liberal brindó a los médicos capacidad para determinar los estándares de su práctica así como obtener autonomía técnica respecto a otros grupos profesionales. Lo anterior fue desarrollado con el objetivo de no someterse a los mecanismos de racionalización de la produción de servicios vinculados a la búsqueda de la eficiencia en el uso de los recursos y el control de los costos de la atención en las instituciones.

Los mecanismos específicos a través de los cuales la profesión médica logró el estatus profesional se encuentran íntimamente ligados a su relación con los diversos agentes, siendo el más importante de ellos, de nueva cuenta, el Estado. Es este último el que otorga o no a los grupos profesionales la capacidad de regular su propia práctica. Los resultados de ese proceso han sido distintos en cada país y distintos modelos de regulación profesional han sido desarrollados. En un extremo se encuentran aquellos modelos en que el Estado mantiene el control, por medio de sus agencias, sobre las modalidades de la producción de médicos, su entrada al mercado, su participación en las instituciones de salud, su distribución geográfica, la forma por la cual los médicos son remunerados y otras características. En el otro extremo se encuentran los modelos donde la profesión es altamente independiente y que tienen amplia capacidad para determinar todos o la mayoría de esos aspectos.

Desde luego, el resto de los agentes que intervienen en la definición de la producción de los servicios ha tenido menor o mayor influencia en el desarrollo de los distintos modelos de regulación profesional. Por ejemplo, en los sistemas de salud donde la participación del Estado no es tan amplia, los financiadores y productores privados de servicios han mostrado cierta capacidad para definir el modelo de regulación de los grupos profesionales. Las reformas de los sistemas de salud que se han gestado en diversos países en los últimos años, y que en muchos sentidos implican el retiro parcial o total del Estado en áreas en las que históricamente ha intervenido, brindan una oportunidad importante para estudiar y entender los mecanismos de regulación y sus posibles cambios a futuro. Si bien en muchos casos, a partir de la reforma, la participación del Estado en la 
producción de servicios se ha reducido, otros agentes, incluída la población consumidora de servicios de salud, luchan por mantener o aumentar su participación en la definición de la práctica profesional a fin de vigilar que los servicios sean producidos com el máximo de calidad y eficiencia en función de la disponibilidad financiera (Gómez-Dantés \& Zorrilla, 1994).

Es de importancia reconocer que los distintos agentes que participan en la producción de servicios de salud lo hacen persiguiendo sus propios objetivos e intereses, que en ocasiones coinciden pero que comunmente difieren. Por ello la capacidad para tomar decisiones tanto en nivel estratégico como en nivel operativo comunmente es el resultado de la habilidad que cada agente tiene para participar en el proceso, de los recursos con los que cuenta y de la capacidad para aprovechar las oportunidades en momentos de definición (Immergut, 1992). Bajo esa perspectiva se ha logrado entender en al gunos países industrializados la relación entre la práctica profesional y el aumento de los costos de producción de los servicios de salud (Stone, 1980).

Sin duda, la profesión médica es un agente con intereses propios en posesión de una amplia gama de recursos, que van desde los técnicos hasta los ideológicos, que normalmente son puestos en juego al momento de defender sus intereses. En algunos casos el Estado ha logrado dominar el proceso de toma de decisiones pero ello no quiere decir que lo haga sin involucrarse en un proceso de negociación (WiIliamson, 1989). En general los agentes financieros, incluído el Estado cuando juega ese papel, son "dependientes" de los médicos como productores directos, al ser la profesión el único grupo autorizado, legal y socialmente, para tomar un sinnúmero de decisiones técnicas para la prestación de los servicios. Un componente fundamental en la racionalización de las decisiones en la producción de los servicios, dentro de las propuestas de reforma sigue siendo la capacidad que tienen los médicos para tomar tales decisiones en el nivel operativo.

El estudio de los modelos de regulación se ha llevado a cabo ampliamente en los países industrializados de América y Europa. En diversos casos los estudios han tomado un carácter comparativo lo cual, de acuerdo a sus autores, ha permitido entender en mayor profundidad las características de cada caso particular (Garpenby, 1990; Wilsford, 1991). El interés por estudiar el papel de los médicos en los sistemas de salud y en consecuencia los distintos modelos desarrollados para su regulación se ha extendido a otros países. En un libro reciente editado por Hafferty \& Mckinlay (1993), se presentan casos de diversos países como Estados Unidos, Inglaterra, Francia y Canadá que han sido ampliamente documentados. A ellos se suman los de la Federación de Estados Independientes, la ex-Checoslovaquia, China y Grecia, que han sido escasamente referidos a la literatura especializada. Llama la atención que los editores señalan la ausencia de estudios de la profesión médica en otras regiones del mundo, particularmente en América Latina, lo cual es atribuido principalmente a la incapacidad de identificar grupos de investigación que se hayan especializado en el estudio del tema.

Es de destacar que una consecuencia de la reforma de los sistemas de salud es el renovado interés que existe por el estudio de las profesiones de la salud desde los distintos enfoques. Si bien existen estudios antecedentes en América Latina, su aplicación, sistematización y actualización son necesarias ya que sus análisis nos deben llevar a entender los aspectos fundamentales, tanto explícitos como implícitos, de las modalidades de participación de los distintos grupos profesionales dentro de la re-estructuración de los sistemas de salud.

\section{La relación Estado-profesión}

El desarrollo de un mecanismo de regulación profesional es producto de un proceso histórico donde el estudio de las formas de interacción que existen entre la profesión y el Estado es preponderante. Para los propósitos de este trabajo se considera que el Estado tiene influencia en dos niveles: en la regulación de las relaciones sociales y económicas en general y en su participación en el sistema de salud.

En la actualidad, en muchos países de América Latina, el Estado tiende a redefinir su papel. A la luz de los hechos, parece ser que, tanto en el papel económico como en el político-social, se avecinan grandes cambios. La estratégia de crecimiento que priorizaba el apoyo al capital interno - que fue compartida por distintos países de la región a partir de la postguerra, generó importantes niveles de crecimiento en varios de esos países de América Latina y terminó con la crisis de la deuda externa en los años 80 -, ha sido sustituída en la actualidad por una política económica que tiende a la integración de grandes bloques comerciales internacionales. Paralelamente el Estado tiende a tomar más el papel de árbitro de las relaciones comerciales y económicas que él de interventor, y su retiro de la producción directa es evidente (Picó, 1990). 
Paralelamente a esos cambios en el nivel macro, también se está llevando a cabo la redefinición del Estado como proveedor de servicios sociales y asistenciales. Por años el Estado jugó un papel dinámico en las transformaciones del sistema de salud. Un aspecto importante de ello fue la creación de los sistemas de seguridad social en la región bajo su control administrativo, teniendo como objetivo principal la restitución de la fuerza de trabajo involucrada en el sector formal de la economía. En el campo de la salud, la reforma actual de los sistemas sanitarios en muchos países de América Latina refleja la redefinición del papel del Estado. Ese cambio busca responder a las necesidades de la nueva estrategia de desarrollo económico vía la apertura de los mercados internos. Como consecuencia de lo anterior existe un proceso de reacomodo de la relación entre el Estado y el resto de los agentes que participan en la producción de servicios de salud, incluidos entre ellos los trabajadores de la salud.

No obstante, no todas las categorías del personal de salud se encuentran en la misma posición para obtener mayores beneficios de este reacomodo. Se sabe por diversos estudios que los médicos en particular se encuentran en posición privilegiada en la toma de decisiones en el nivel operativo y, muchas veces, en el nivel estratégico del sistema. Esto les ha permitido ser partícipes de la definición de los modelos de regulación de los cuales son sujetos. Los modelos de regulación se definen básicamente a partir de las características de la participación de los profesionales como productores de servicios de salud.

\section{El modelo de regulación}

Al tratar de entender los mecanismos definidos para regular la participación de los profesionales en la producción de servicios de salud, hay que tomar en consideración las propuestas planteadas en la literatura. Una propuesta que parece apropiada como punto de partida es la elaborada por Moran \& Wood (1993). Estos autores definen el modelo de regulación como el mecanismo utilizado para definir las características de la práctica y los estándares técnicos que ésta tiene que alcanzar. Moran y Wood consideran que el modelo de regulación se compone de las siguientes variables: a) los mecanismos de entrada al mercado (incluidos licenciamiento y certificación), b) el control de la competencia profesional, c) la estructura del mercado de trabajo y d) el mecanismo de pago.
Cada uno de estos aspectos se describe a continuación.

a) Control del mercado. Este aspecto señala la forma en la que se regula la entrada al mercado de los individuos habilitados para ejercer como médicos. Para ello normalmente se encuentran estipuladas las condiciones que dichos individuos deben cumplir para su ingreso. Debido a la alta complejidad que corresponde al trabajo de un médico, los mecanismos de entrada al mercado suelen ser sumamente sofisticados para definir quién y bajo que circunstancias se puede practicar medicina. La estrategia más común para controlar la entrada al mercado es el establecimiento de un mecanismo de licenciamiento a través del cual se otorga la autorización legal, después de cumplir con ciertos requisitos tales como escolaridad, la pertenencia a asociaciones profesionales y otros, a todos aquellos aspirantes a ingresar al mercado.

b) Regulación de prácticas de competencia. Este aspecto de la regulación suele estar relacionado a la competencia que otros grupos profesionales hacen a los médicos. Dado que los médicos suelen tener un virtual monopolio del conocimiento que es aplicado en la producción de servicios de salud, el acceso de otros grupos a ese conocimiento puede ser regulado tanto en el nivel educativo como en el de la producción misma de los servicios, en las instituciones de salud. Así existen mecanismos legal es o extra-legales (ideológicos) para regular la competencia de otros modelos de práctica médica como son la homeopatía o la herboIaria. La competencia, no obstante, también puede ser regulada entre los propios miembros de la profesión. Por ejemplo, en algunos países existen reglamentaciones sobre la prohibición o permisividad en el uso de propaganda para la promoción de los servicios que otorgan los practicantes.

c) Estructura del mercado de trabajo. Existen distintas formas en las que los médicos se integran al mercado de trabajo para producir servicios. En países donde el sector público es dominante, los médicos suelen ser asalariados en un alto porcentaje. No obstante, siempre existe un sector del mercado donde los médicos producen servicios de manera privada, y es común que lo hagan sin estar vinculados a alguna institución. Dentro del sector privado existen interacciones entre médicos para buscar reducir sus costos de operación. En muchos casos los médicos pueden combinar la práctica asalariada, pública o privada, con la liberal. En cada país el volumen de asalariados, de médicos liberales y de aquellos que combinan ambas 
prácticas varía, como tambiém lo hacen aquellos médicos que no producen servicios ya sea porque lleven a cabo otro tipo de actividades o porque no encuentren la forma de vincularse a la producción de servicios. Dentro de la estructura del mercado se consideran también los patrones de distribución geográfica de los médicos, los cuales pueden estar determinados por una legislación específica o por mecanismos definidos por el Estado para incentivar cierto tipo de distribución. Las características del proceso de trabajo forman también parte de la estructura del mercado. En años recientes las instituciones han puesto en práctica diversos mecanismos para buscar que la producción de servicios reduzca sus costos y incremente sus niveles de eficiencia y calidad, lo cual afecta la capacidad que esas instituciones tienen para emplear médicos.

d) Mecanismo de pago. La forma preferida de los médicos para percibir sus ingresos es a través del pago por servicio. No obstante, a nivel mundial, el mecanismo más difundido es el asalariamento. En muchos países la profesión ha tenido la capacidad de hacer valer sus preferencias sobre la forma en la que se lleva a cabo su remuneración. Dentro de un mismo país las instituciones públicas pueden pagar salarios distintos a los médicos. En años recientes las estrategias para incentivar la productividad y la calidad a través del mecanismo de pago se han expandido, no sin disputas entre la profesión y las instituciones sobre la mejor forma de lograr ese objetivo.

El conjunto de estos cuatro aspectos constituye el modelo de regulación. Para Moran \& Wood (1993), existen tres variantes regulatorias principales. La primera se denomina auto-regulación y está caracterizada por permitir a los propios profesionales definir los mecanismos de entrada al mercado y de competencia. La segunda se denomina auto-regulación bajo sanción estatal y se caracteriza por que las ins- tituciones encargadas de formular y implementar el mecanismo de regulación (normalmente compuestas por los propios profesionales) actúen con el consentimiento y apoyo del Estado. Finalmente la última variante es la regulación directa del Estado. En ella la autoridad para regular descansa directamente en el Estado o en sus agencias. Así la regulación se lleva a cabo por instituciones públicas especializadas en dicha función (Figura 1).

La aplicación de esta propuesta a distintos países debe considerar su adaptación en cada uno de ellos. Si bien la propuesta parece incorporar las áreas más importantes en las que la regulación se lleva a cabo, su orientación económica no incorpora apropiadamente el contenido político de la interacción. De esta forma habría que analizar, por ejemplo, la existencia o ausencia de agrupaciones o asociaciones profesionales y el papel que juegan en la definición del papel de los médicos en las distintas áreas del modelo. En los hechos, las asociaciones profesionales juegan un doble papel ya que, por un lado, su existencia misma puede ser regulada por el Estado (o otros actores) y al mismo tiempo pueden funcionar como agentes reguladores.

\section{Redefiniendo el objeto de estudio}

El estudio de las profesiones de salud desde distintos enfoques teóricos ha mantenido una gran dinámica en los últimos años. Ello ha llevado los frentes en discusión a cuestionarse no sólo los instrumentos con los que se pretende llevar a cabo el análisis de la realidad sino el objeto mismo que se está estudiando. Si bien la noción del modelo de regulación nos permite acotar una parte del objeto de estudio, éste no se agota en la definición del modelo mismo. El debate en la actualidad empieza a desprenderse claramente de la discusión sostenida en tor-

\section{Figura 1}

Tres modelos regulatorios. Areas de regulación.

\begin{tabular}{|l|l|l|l|l|}
\hline & $\begin{array}{l}\text { ingreso al } \\
\text { mercado }\end{array}$ & $\begin{array}{l}\text { prácticas } \\
\text { competitivas }\end{array}$ & $\begin{array}{l}\text { estrutura del } \\
\text { mercado }\end{array}$ & remuneración \\
\hline auto-regulación & & & & \\
\hline con sanción estatal & & & & \\
\hline regulación estatal & & & & \\
\hline
\end{tabular}

Fuente: Moran \& Wood (1993). 
no al modelo propuesto por Eliot Freidson hace ya más de 20 años. Hoy día se empieza a cuestionar la distinción misma del binomio Estado-profesión (médica) sobre la cual están basados prácticamente todos los modelos existentes.

Según Johnson (1995), la propuesta que ha dominado la discusión durante este periodo, en la que se define al Estado y a la profesión como dos entidades separadas donde el primero aparece extendiendo sus aparatos de control a toda la sociedad mientras que la segunda se presenta en la búsqueda permanente por autonomía, a perdido sentido a la luz de análisis alternativos. Retomando algunos conceptos propuestos por Foulcault como normalización y gobernamentalidad, Johnson propone que tal separación no existe si se considera que, en todos aquellos países donde la provisión de servicios se realiza en forma socializada, la profesión médica se ha desarrollado como parte del Estado mismo. El concepto de gobernamentalidad se refiere al desarrollo de un tipo de gobierno asociado a institucionalización de conocimientos específicos y su uso en la interpretación de la realidad social. El proceso histórico por lo cual se ha llegado a esa situación permite entender como la creación del Estado y el desarrollo de un conocimiento experto generado por los profesionales se encuentran íntimamente ligados. Posteriormente el conocimiento experto se convierte en condición para el control social y el ejercicio del poder político. En el área de la salud, el éxito de los profesionales médicos para construir una realidad social con validez universal se podría interpretar como una consecuencia del reconocimiento oficial de su papel de expertos.

Por otra parte Light (1995), al referirse a la relación entre Estado y profesión, prefiere hablar de modelos de control basados en el supuesto de que dicha relación rebasa el concepto llano de una relación de mercado entre empleadores y empleados, o entre productores y consumidores. De hecho instala la relación en un marco político donde los grupos involucrados se encuentran en proceso continuo y constante de bal anceo de poderes. El componente histórico en la propuesta de Light es básico. Propone que el modelo de control es consecuencia de los cambios cualitativos que se dan en la interacción entre los actores participantes en un periodo de tiempo. Sólo así es posible entender, por ejemplo, la posición de fuerza que actualmente goza la profesión médica en Alemania, siendo que, a principios del siglo, su posición frente a los agentes finacieros y reguladores era de suma debilidad. La política sanitaria durante el nazismo contó con el apoyo de la profesión lo cual permitió a ésta consolidar su estatus profesional, incrementar su capacidad de negociación respecto a otros agentes y en consecuencia obtener una posición de fuerza en la arena política (Light \& Levine, 1988). A partir de otros hechos, en la actualidad, la posición de fuerza de la que goza la profesión médica en ese país se refleja en su capacidad económica y en el prestigio social obtenido.

Para Light la relación profesión-Estado, y por tanto el modelo de control, se establece sobre dos ejes principales. El primero de ellos gira en torno al concepto freidsoniano de dominio que, en su versión original, significa no sólo el control sobre el trabajo técnico sino también sobre los aspectos financieros, las estructuras institucionales, los poderes alternos, el carisma cultural y incluso sobre la construcción de la realidad social. El segundo gira en torno a la ubicación de los médicos en el mercado laboral. Se conjugan por lo tanto un criterio político-social con uno de tipo económico. En uno de los extremos del primer eje aparece el dominio profesional mientras que, en el extremo contrario, lo hace el dominio del Estado. Ejemplo claro del primer caso es la profesión médica norteamericana, mientras que del segundo lo es la profesión médica en la ex-Unión Soviética la cual carecía de control sobre aspectos financieros, distribución de recursos y división del trabajo de los profesionales de salud.

La ubicación en el mercado de trabajo varía también entre dos extremos. Uno de ellos está representado por una profesión cuyos miembros son pequeños empresarios que se emplean a sí mismos, o lo que se conoce como práctica liberal. En el extremo contrario, los miembros de la profesión se encuentran en condición asalariada ya sea por instituciones públicas o privadas. Entre ambos extremos se encuentran una gran cantidad de variantes, especialmente cuando los médicos son capaces de combinar trabajos en el sector público y en el sector privado.

\section{Discusión}

Es por demás interesante utilizar las propuestas aquí reseñadas para identificar y analizar las relaciones existentes entre el mercado de servicios de salud y el papel que los profesionales juegan en él, en la actualidad. De entrada debe entenderse que las relaciones entre los agentes que participan en este mercado no son sólo económicas sino también políticas (Figu- 


\section{Figura 2}

Diferencias entre el enfoque económico y político en el análisis de la participación de la profesión médica en la producción de servicios de salud.

\begin{tabular}{|l|l|l|l|}
\hline enfoque & definición & objetivos & campo de acción \\
\hline económico & agente económico & $\begin{array}{l}\text { oferta de servicios } \\
\text { asesoría al paciente }\end{array}$ & mercado \\
\hline político & actor político & $\begin{array}{l}\text { búsqueda de autonomía/ } \\
\text { defensa de intereses profesionales }\end{array}$ & estructura de poder \\
\hline
\end{tabular}

ra 2). Recientemente en muchos países industrializados se ha buscado contrarrestar el poder de los profesionales a través de diversas estrategias. Autores como Klein (1989) y Freddi \& Bjorkman (1989) han señalado el gran poder desarrollado por los médicos en los sistemas sanitarios públicos de Europa así como los mecanismos puestos en práctica por los agentes financieros para lograr su control. Uno de ellos es la llamada competencia manejada que intenta simular las condiciones de un mercado no regulado a fin de obtener la mejor relación entre precio y eficiencia en los servicios y en última instancia lograr el control de los costos, todavía bajo un claro control estatal.

No obstante, una de las dificuldades más importantes en las que se enfrentan los mercados de servicios está relacionada a la naturaleza compleja y esotérica del trabajo de los profesionales. Esto determina en gran medida las imperfecciones y las fallas de los mercados. Los "productos" que ofrecen los profesionales, sus cualidades y sus costos son frecuentemente difíciles de medir. Dado el bajo nivel de información que los consumidores poseen, para ellos es una difícil tarea hacer la selección del mejor proveedor en función del precio y de la calidad que éste ofrece. En última instancia el consumidor tiene que confiar en la capacidad del proveedor muchas veces en base a actos de fé y no sobre una base de información perfecta. En consecuencia el mercado de servicios de salud, además de sus connotaciones económicas, involucra otras de tipo social, cultural y político. Se puede decir que muchas de las llamadas imperfecciones del mercado "en realidad son patrones de interacción determinados social y culturalmente que fallan (o son imperfectos) en el sentido de que no caben en los estrechos modelos económicos" (Light, 1995:39).

Finalmente debe señalarse que los esfuerzos por regular o controlar el trabajo de los profesionales se encuentran hoy en un profundo periodo de redefinición. Entre otras variables relacionadas con ese proceso encontramos el papel que juega hoy el Estado en la producción de servicios de salud, el incremento de la participación de otros agentes y la permanente necesidad por racionalizar el uso de los recursos disponibles y aumentar la eficiencia y la calidad de los servicios. En sus primeras etapas este proceso de redefinición parece estar minando la capacidad de los profesionales en tomar decisiones al tiempo que otros agentes ganan terreno. No obstante es temprano para señalar las verdaderas consecuencias. Sólo el análisis continuo de dicha realidad nos permitirá obtener las respuestas buscadas. 


\section{Agradecimientos}

El apoyo financiero de la Organización Panamericana de la Salud fue importante para la redacción del presente trabajo. La OPS organizó una reunión de trabajo en Rio de Janeiro, en febrero de 1995, donde un grupo de investigadores de cinco países de América Latina con experiencia en investigación de recursos humanos para la salud revisó, criticó y atualizó los conceptos que sirvieron de base para la redacción del trabajo con el fin último de dar origen a un proyecto multinacional sobre los mecanismos de regulación de la profesión médica. No obstante, todos los puntos de vista expuestos en el presente texto son responsabilidad absoluta de sus autores.

\section{Referencias}

ACADEMIA NACIONAL DE MEDICINA, 1993. LosServicios de Salud y el Tratado de Libre Comercio. México: Academia Nacional de Medicina.

FREDDI, G. \& BJORKM AN, J., 1989. Controlling Medical Professionals. London: Sage

GARPENBY, P., 1990. The State and the Medical Profession: A Cross-National Comparison of the Health Policy Arena in the UK and Sweden.19451985. Linkopig Studies in Arts and Sciences.

GOMEZ-DANTES O. \& ZORRILLA P.,1994. La Protección del Consumidor en el Campo dela Salud. Serie Economía y Salud. M éxico: Fundación Mexicana de la Salud.

HAFFERTY, F. \& M CKINLAY, J., 1993. The Changing Medical Profession. An International Perspective. New York: Oxford University Press.

IM MERGUT, E., 1992. Health Politics: Interests and Institutions in Western Europe. New York: Cambridge University Press.

JOHNSON, T., 1995. Health Professions in Europe. London: Routledge.

KLEIN, R., 1989. The Politics of the NHS. 2nd Edition. London: Longman.

LIGHT, D., 1995. Countervaling powers: In: Health Professions in Europe (Johnson T., Larkin G. y Saks, M., eds.) pp. 25-41. London: Routledge.
LIGHT, D. \& LEVINE, S., 1988. The changing character of the medical profession. A theoretical overview. The Milbank Quarterly, 66(Suppl. 2):1-9.

MORAN, M. \&WOOD, B., 1993. States, Regulation and the Medical Profession. Buckinghan: Open University Press.

NARRO, J. \& M OCTEZUMA, B. J. C., 1992. La Seguridad Social y el Estado Moderno. México: Instituto Mexicano del Seguro Social, Fondo de Cultura Económica, Instituto de Seguridad y Servicios Sociales para los Trabajadores del Estado.

PICO J., 1990. Teorías sobre el Estado de Bienestar. Madrid: Siglo Veintiuno Editores.

STONE, D., 1980. The Limits of Professional Power: National Health Care in Federal Republic of Germany. Chicago: University of Chicago Press.

WILDING, P.,1982. Professional Power and Social Welfare. London: Routledge \& Kegan Paul Ltd.

WILLIAMSON, P., 1989. Corporatism in Perspective. An Introduction Guide to Corporatist Theory. London: Sage Publications.

WILSFORD, D., 1991. Doctors and the State: The Politics of Health Care in France and the United States. Durham: Duke University Press. 